九州大学学術情報リポジトリ

Kyushu University Institutional Repository

A spatiotemporal signature of cortical pain relief by tactile stimulation : An MEG study

早水，真理子

https://doi.org/10.15017/1785363

出版情報: 九州大学，2016，博士（医学），課程博士 バージョン：

権利関係 : 全文ファイル公表済 


\title{
A spatiotemporal signature of cortical pain relief by tactile stimulation: An MEG study
}

\author{
Mariko Hayamizu a,b,*, Koichi Hagiwara a , Naruhito Hironaga a , Katsuya Ogata a , \\ Sumio Hoka ${ }^{\text {b }}$, Shozo Tobimatsu ${ }^{\text {a }}$ \\ a Department of Clinical Neurophysiology, Neurological Institute, Faculty of Medicine, Graduate School of Medical Sciences, Kyushu University, Fukuoka, Japan \\ ${ }^{\mathrm{b}}$ Department of Anesthesiology and Critical Care Medicine, Graduate School of Medical Sciences, Kyushu University, Fukuoka, Japan
}

\section{A R T I C L E I N F O}

\section{Article history:}

Received 18 November 2015

Accepted 31 January 2016

Available online 12 February 2016

\section{Keywords:}

Intra-epidermal electrical stimulation

Mechanical tactile stimulation

Tactile-induced analgesia

Insular-opercular region

Magnetoencephalography

\begin{abstract}
A B S T R A C T
Recently, the cortical mechanisms of tactile-induced analgesia have been investigated; however, spatiotemporal characteristics have not been fully elucidated. The insular-opercular region integrates multiple sensory inputs, and nociceptive modulation by other sensory inputs occurs in this area. In this study, we focused on the insular-opercular region to characterize the spatiotemporal signature of tactile-induced analgesia using magnetoencephalography in 11 healthy subjects. A $\delta$ (intra-epidermal electrical stimulation) inputs were modified by $A \beta$ (mechanical tactile stimulation) selective stimulation, either independently or concurrently, to the right forearm. The optimal inter-stimulus interval (ISI) for cortical level modulation was determined after comparing the 40-, $60-$, and 80 -ms ISI conditions, and the calculated cortical arrival time difference between $A \delta$ and $A \beta$ inputs. Subsequently, we adopted a 60-ms ISI for cortical modulation and a 0-ms ISI for spinal level modulation. Source localization using minimum norm estimates demonstrated that pain-related activity was located in the posterior insula, whereas tactile-related activity was estimated in the parietal operculum. We also found significant inhibition of pain-related activity in the posterior insula due to cortical modulation. In contrast, spinal modulation was observed both in the posterior insula and parietal operculum. Subjective pain, as evaluated by the visual analog scale, also showed significant reduction in both conditions. Therefore, our results demonstrated that the multisensory integration within the posterior insula plays a key role in tactile-induced analgesia.
\end{abstract}

(c) 2016 Elsevier Inc. All rights reserved.

\section{Introduction}

The analgesic effect of vibrotactile stimuli has been acknowledged as an important mechanism that can modulate pain perception (Melzack and Wall, 1965). Practically, people unconsciously rub a painful part of their body to reduce pain. Several studies have shown the analgesic effect of both tactile and vibratory stimuli in psychophysiological evaluation in healthy subjects (Mancini et al., 2014; Yarnitsky et al., 1997) and in chronic pain conditions (Lundeberg et al., 1984; Roy et al., 2003; Staud et al., 2011). Additionally, an electrophysiological study with laser-evoked potentials demonstrated the attenuation of a vertex component by concurrently applied vibratory stimulation (Kakigi and Shibasaki, 1992). Because these studies presented nociceptive and non-nociceptive stimuli simultaneously, cross-modal interaction most likely occurred in the spinal cord. On the contrary, recent somatosensory evoked potential (SEP) and magnetic field (SEF) studies (Inui et al., 2006; Mouraux and Plaghki, 2007; Testani et al., 2015) have indicated

\footnotetext{
* Corresponding author at: Department of Clinical Neurophysiology, Neurological Institute, Faculty of Medicine, Graduate School of Medical Sciences, Kyushu University, 3-1-1 Maidashi, Higashi-ku, Fukuoka 812-8582, Japan. Fax: + 81926425545.

E-mail address: hayamizu@med.kyushu-u.ac.jp (M. Hayamizu).
}

a supraspinal mechanism in tactile-induced pain relief via experimental paradigms with concurrent bimodal inputs at the cortical level. However, the precise spatiotemporal profile for centrally mediated tactileinduced analgesia is largely unknown.

To date, several neuroimaging studies have demonstrated that the insular-opercular region (IOR) is consistently involved in pain processing (Apkarian et al., 2005; Peyron et al., 2000, 2002). Some reports have also suggested that this region encodes quantitative as well as qualitative aspects of pain (Iannetti et al., 2005; Oertel et al., 2012; Timmermann et al., 2001). Furthermore, the IOR is known to be involved in multisensory processing, including tactile sensation, pain, proprioception, temperature, and vestibular information (Mazzola et al., 2012; zu Eulenburg et al., 2013). Thus, we hypothesized that the IOR is a key site for multisensory interactions, possibly generating tactileinduced analgesia at the cortical level.

In the present study, we sought to elucidate the cortical representation of pain relief in a temporally and spatially precise manner. We adopted mechanical tactile stimulation (MTS) and intra-epidermal electrical stimulation (IES) for selective $A \beta-A \delta$ interaction. MTS has higher selectivity for A $\beta$ fibers (Onishi and Kameyama, 2011), and this is suitable for increasing the sensitivity of source separation between tactilerelated and pain-related activity (Baumgärtner and Treede, 2009). IES 
is well known as a selective Aס stimulation technique (Inui et al., 2002; Inui and Kakigi, 2012; Mouraux et al., 2010). Then, we recorded magnetoencephalographic (MEG) responses with minimum norm estimates (MNE) analysis, which is widely used as a distributed source analysis method enabling better delineation of spatial and temporal features. With respect to spatial localization, it is crucial to determine the three dimensional segregation within the IOR, because this area has been subdivided into cytoarchitectonically-distinct areas harboring multisensory representations in fMRI studies (Eickhoff et al., 2006a,b; Kurth et al., 2010; Mazzola et al., 2012; zu Eulenburg et al., 2013). Furthermore, an SEF study with multidipole analysis demonstrated that an IES-evoked SEF response in the insula was localized more anteriorly compared with a transcutaneous electric SEF response (Inui et al., 2003). From several lines of evidence, we decided to assess the spatiotemporal signature of tactile-induced analgesia in the IOR.

\section{Methods}

\section{Subjects}

Eleven healthy right-handed volunteers participated in this study. The subjects had no history of neurological conditions or chronic pain. They also had no current or prior history of traumatic events or surgery involving the right arm, and were not on any medication. Written informed consent was obtained from all subjects. This study was approved by the Institutional Ethics Committee of Kyushu University.

\section{$A \delta$ and $A \beta$ stimulation}

We delivered noxious pain stimuli by IES with the aim of selectively stimulating A $\delta$ fibers (Inui et al., 2002; Inui and Kakigi, 2012; Mouraux et al., 2010). The stimulus consisted of double current pulses separated by a 10 -ms interval, and each pulse had a triangular waveform with a 0.5 -ms rise and 0.5-ms fall, as per previous reports (Inui and Kakigi, 2012; Omori et al., 2013). The pain intensity was adjusted individually for each subject prior to the experiment. We aimed to produce a definite, but tolerable pinprick sensation, corresponding to $20-40 / 100$ on a visual analog scale (VAS). The VAS range was presented as a horizontal line, which was anchored by "no pain" (score of 0 ) and "worst imaginable pain" (score of 100), where a score of 5-44 indicates mild pain (Hawker et al., 2011). Stimulus intensities were set lower than $0.3 \mathrm{~mA}$ to minimize the possibility of concurrent $A \beta$ fiber stimulation. Mean stimulus intensity for IES was $0.26 \pm 0.03 \mathrm{~mA}$.

For the innocuous tactile stimulation, we used piezoelectric actuators (KGS, Saitama, Japan) to control the MTS. This enabled us to produce time-locked A $\beta$-specific afferent inputs (Onishi et al., 2010). The stimulus probe had eight pins, and each pin protruded $0.7 \mathrm{~mm}$ with a maximum pushing force of $0.33 \mathrm{~N} /$ pin, producing a sensation that was almost identical to real touch. The mechanical onset delay from the trigger to the highest pin position was less than $0.5 \mathrm{~ms}$, and the stimulus duration was $10 \mathrm{~ms}$. We delivered white noise binaurally throughout the experiment to mask stimulator noise.

\section{Experimental paradigm}

IES and MTS were delivered to the right forearm, slightly distal from the elbow. The stimulation points were located in close proximity $(<1 \mathrm{~cm})$ to one another so that the inputs from both modalities occurred within the same dermatome. We expected that this would maximize the effect of the tactile analgesia (Mancini et al., 2014). Before beginning the experiment, we estimated the cortical arrival times of the $A \beta$ and $A \delta$ inputs using the known conduction velocities (CVs) of each fiber and the distance from the forearm to the $\mathrm{C} 1$ vertebra of the spine (Inui et al., 2006). The average distance between the forearm and the spinal cord (i.e., the $\mathrm{C} 7$ vertebra of the spine) was approximately $50 \mathrm{~cm}$, and that between $\mathrm{C} 7$ and $\mathrm{C} 1$ was approximately $10 \mathrm{~cm}$. Given that the CV of the A $\delta$ fiber is $10-15 \mathrm{~m} / \mathrm{s}$ (Kakigi and Shibasaki, 1991) and that of the $A \beta$ fiber is $60 \mathrm{~m} / \mathrm{s}$ (Inui et al., 2006), we estimated that it

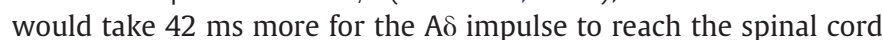
(we adopted the slowest CV of the A $\mathrm{d}$ fiber, i.e., $10 \mathrm{~m} / \mathrm{s}$ for the calculation). Similarly, the CVs of the spinothalamic tract and the posterior column are $8-10 \mathrm{~m} / \mathrm{s}$ and $50-60 \mathrm{~m} / \mathrm{s}$ (Kakigi and Shibasaki, 1991), respectively. Therefore, we estimated the difference in conduction time within the spinal cord to be $11 \mathrm{~ms}$. Thus, we predicted that the cortical arrival time of the A $\delta$ projection would be $53 \mathrm{~ms}$ later than that of $\mathrm{A} \beta$. We also conducted an experiment to estimate the $\mathrm{CV}$ of the $\mathrm{A} \delta$ fiber in three subjects by stimulating the right dorsal hand and shoulder using IES.

Because the inter-stimulus interval (ISI) between IES and MTS was critical in this study, we performed two experiments to determine the optimal ISI for cortical level modulation (Exp. I) and to assess cortical modulation at a fixed ISI (Exp. II). In Exp. I, we adopted four different stimulus conditions: a) control IES, b) control MTS, c) 0-ms ISI (IES and MTS were applied simultaneously), and d) three types of ISI settings (Fig. 1). Three different ISI settings (40-, 60-, or 80-ms) were tested to determine the optimal ISI for the most effective suppression at the supraspinal level. Seven subjects (three females; age, 25-49 years) participated in this experiment. The experiment comprised five sessions, and each session was separated by 3 to 4 min of rest to avoid any attenuation of responses as well as habituation of the subjective pain sensation. Ten trials were performed for each condition in each session. Therefore, each session consisted of 40 randomly ordered trials with inter-trial intervals of 4 to $5 \mathrm{~s}$. According to the results of Exp. I (see Results), we used the 60-ms ISI condition for the cortical inhibitory condition in Exp. II (Fig. 1d). Thus, the 0-ms ISI (IES and MTS were applied simultaneously) was designed to determine interaction at the spinal level, while the 60-ms ISI was formulated to elucidate the presence of tactile-induced analgesia at the supraspinal level. The seven subjects from Exp. I and four further subjects were recruited for Exp. II. Thus, a total of 11 subjects (four females; age, 21-49 years) were examined. The subjects rated subjective pain intensities for each condition using a VAS prior to MEG measurements. Twenty trials (five trials for each condition) were randomly delivered using the same inter-trial intervals, and the subjects gave a VAS score for each individual stimulus.

\section{Data acquisition}

SEFs were recorded using a 306-channel whole-head MEG system (Neuromag; Elekta, Helsinki, Finland). Prior to recording, we attached four head position indicator (HPI) coils to the scalp, and used a 3D digitizer (Fastrack; Polhemus, Colchester, VT, USA) to measure the anatomical landmarks (nasion and left and right pre-auricular points), the HPI positions, and the head surface. We conducted HPI measurement immediately before the first session, and the subject maintained their initial head position throughout the recording. The subjects were seated in an upright position and instructed to keep their eyes open. Their vigilance was carefully monitored by a video camera positioned in a shielded room. The MEG signals were digitally sampled at $1000 \mathrm{~Hz}$ with a band-pass filter of $0.3-330 \mathrm{~Hz}$. Structural MRI was obtained using a 3.0-T high resolution MRI scanner (Achieve; Philips N.V. Eindhoven, The Netherlands) for MEG-MRI co-registration and subsequent source localization analysis (repetition time $8.2 \mathrm{~ms}$; echo time $3.8 \mathrm{~ms}$; flip angle $8^{\circ}$; 190 sagittal slices; $1.0 \mathrm{~mm}$ isotropic voxels with no gap).

\section{Data analysis}

We processed all data sets using the spatiotemporal signal separation method (Taulu et al., 2004) to filter out noise signals from outside of the head. We applied a bandpass filter in the 1-57 Hz range, and trials above a rejection threshold of $5000 \mathrm{fT} / \mathrm{cm}$ for gradiometer channels were removed before averaging. The artifact-free data were averaged based on the onset of each stimulus in the control condition, where 
a) Control IES

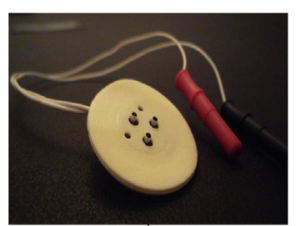

\section{b) Control MTS}

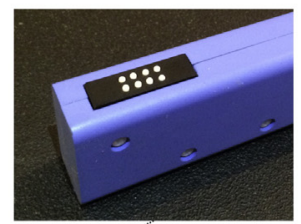

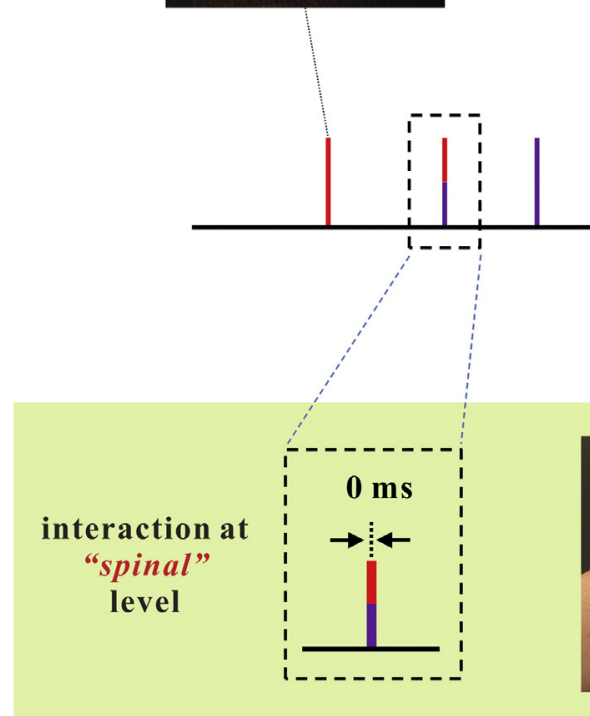

c) 0-ms ISI

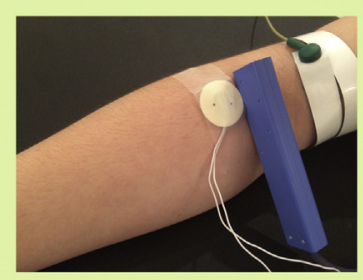

d) 40-, 60-, or 80-ms ISI
Inter-trial interval: 4-5 sec (random)

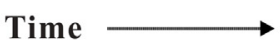

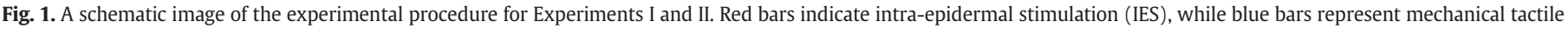

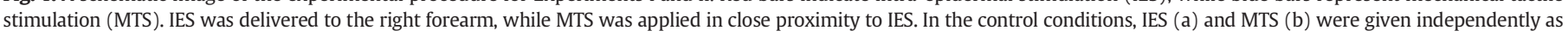

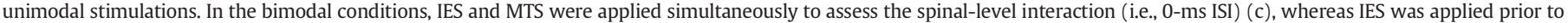
MTS to assess the cortical-level interaction (40-, 60-, or 80-ms ISI) (d). Each of these four conditions was delivered randomly with 4-5 s inter-trial intervals.

averaging was triggered by the onset of IES for each ISI setting (40-, 60-, or 80 -ms). Ten trials multiplied by five sessions (50 trials) were conducted. In Exp. I, 12 data sets [4 conditions ( (a) control IES, (b) control MTS, (c) 0-ms ISI, and (d) delayed-ISI settings (40-, 60-, or 80-ms))] were obtained and group averaged for seven subjects after applying source reconstruction and transformation into the standard brain during source analysis. In Exp. II, four data sets [four conditions ((a) control IES, (b) control MTS, (c) 0-ms ISI, and (d) 60-ms ISI)] were obtained and group averaged for 11 subjects.

Using FreeSurfer software (Dale et al., 1999; Fischl et al., 1999), we reconstructed the cortical surface for each subject using T1-weighted images. We used MNE-suite (http://www.martinos.org/mne/) (Dale and Sereno, 1993; Dale et al., 2000; Hämäläinen and Ilmoniemi, 1994), which is a distributed source model for MEG analysis, to ensure rich spatiotemporal resolution for current sources over the cortical surface in our source localization analyses. The extracted MRI contour was then imported to co-register with the MEG head coordinate system using the scalp surface points obtained by the 3D digitizer. The general procedures for MNE and dynamic statistical parametric mapping (dSPM) (Dale et al., 2000; Molins et al., 2008) were based on our previous studies (Hironaga et al., 2014; Kanamori et al., 2013; Miyaji et al., 2014). For noise normalization in dSPM, we created a noise covariance matrix from -200 to $-100 \mathrm{~ms}$ in the pre-stimulus period. Fig. 2 shows a schematic image of the methodological procedure. First, we defined a region of interest (ROI) for the contralateral IOR on the standard brain (MNI305; provided by FreeSurfer software, MNI = Montreal National Institute (Collins et al., 1994), and then projected this onto each individual brain (Fig. 2a). Within the IOR, the parietal operculum is commonly referred to as the secondary somatosensory cortex (SII) in reference to its functional aspect. Nevertheless, the definition and localization of SII varies from study to study (Eickhoff et al., 2006b), hence we decided to use the parietal operculum as the anatomical description for SII in this study. For optimal spatial segregation between the activities elicited by the control IES and control MTS, we defined a restricted ROI (rROI). Fig. $2 b$ shows the encircled rROI from the activation patterns of group-averaged data. We did this by narrowing down the activated vertices to reveal a region where the dSPM value was larger than $90 \%$ of the highest values in a 20 -ms time window centered at a peak latency:

$r R O I=\left\{v_{i}: d_{S P M}\left(v_{i}\right)>\max _{i, t}\left(d_{S P M}\left(v_{i}, t\right)\right) * 0.9 ; v_{i} \in \mathrm{ROI}, t \in t_{p} \pm 10_{m s}\right\}(1)$

where $v_{i}, d_{S P M}\left(\nu_{i}\right), R O I$, and $t_{p}$ indicate a vertex index, dSPM value of the vertex $\nu_{i}$, the original ROI (i.e., IOR), and a peak latency, respectively. The peak latencies for each modality were initially identified in terms of root-mean-square waveforms for approximately nine pairs of gradiometers located at a contralateral temporal area. This area was first located by visual inspection, and we then confirmed that these latencies corresponded to the peak latencies of source activation curves (source waves) obtained from dSPM values within the original ROI. We used weighted averaging (Hironaga et al., 2014) to determine the center of the vertices within the rROI as follows:

center $=\frac{1}{\sum_{i} d_{S P M}\left(v_{i}\right)} \sum_{i} d_{S P M}\left(v_{i}\right) P\left(v_{i}\right)$

where $P\left(\nu_{i}\right)$ represents the vertex position. After calculating the center coordinates for each modality, we transformed the coordinates in the individual MRIs (expressed in terms of the Right Anterior Superior (RAS) coordinate system) onto the standard brain using the MNITalairach coordinate system. We also created group-averaged MNE 
a) Insular-opercular ROI
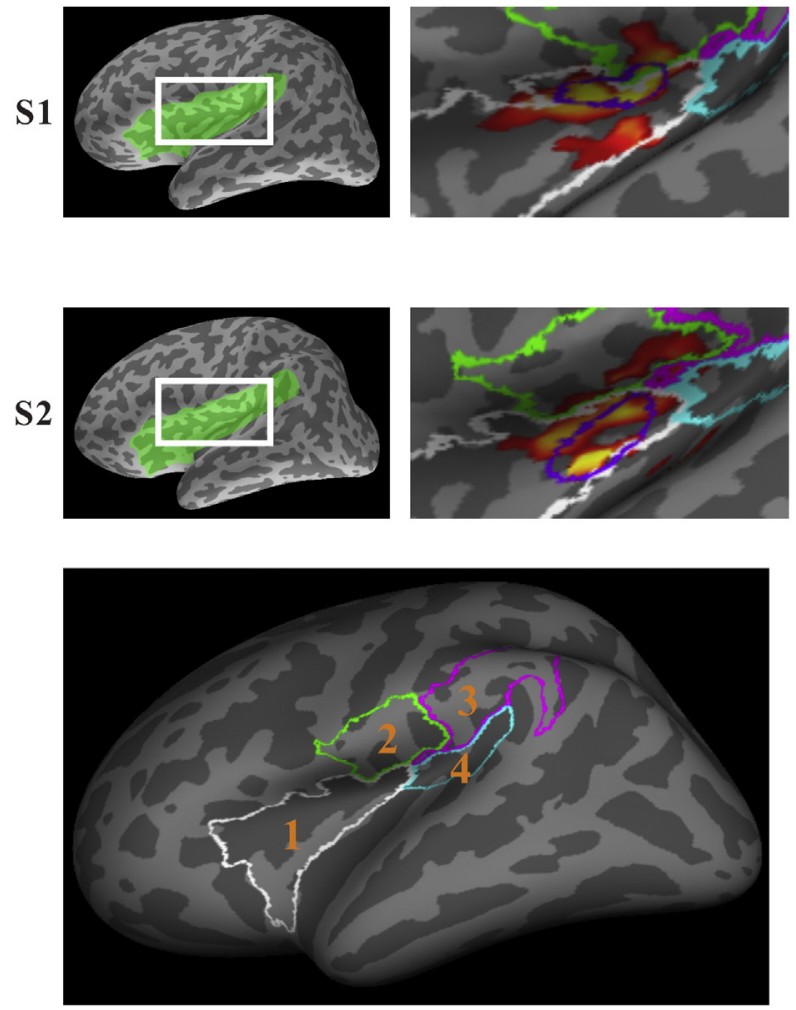

c) Marked rROI
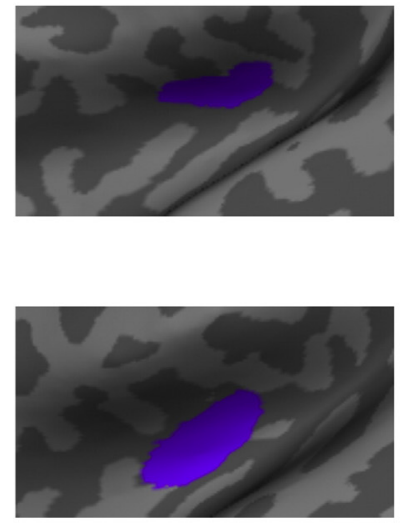

e) Label annotation by FreeSurfer

1) Insula (white)

2) Subcentral cortex (green)

3) Inferior parietal cortex (magenta)

4) Lateral fissure and retroinsular area (cyan)

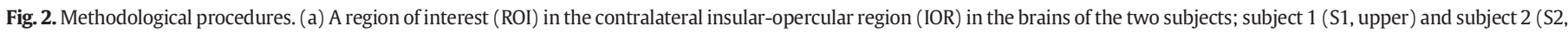

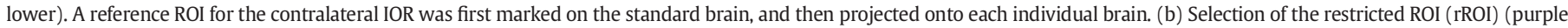

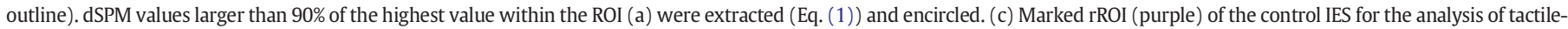

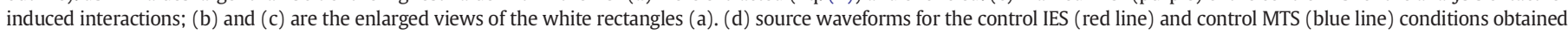

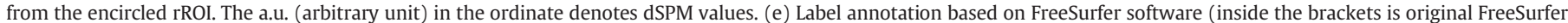

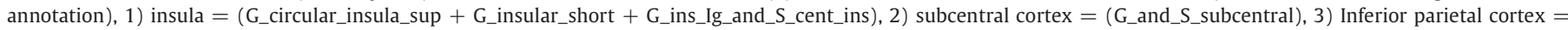
(G_pariet_inf_supramar), and 4 ) lateral fissure and retroinsular area $=$ (Lat_Fis_post).

activation maps after transformation into the standard brain for summed control and actual bimodal conditions with standardization. Standardization by dividing by the maximum values of two conditions, control and bimodal, was performed to retain the activation ratio in each individual. This process was applied for two ISI settings separately.

To investigate tactile-induced interactions in pain processing, we focused on the region most relevant to pain-related activity, namely, the rROI for the control IES. To this end, we marked all activities identified as rROIs for the control IES (Fig. 2c), and reconstructed source waves for each stimulus condition (Fig. 2d). Here, we defined the source wave amplitudes from the 0-, 40-, 60-, and 80-ms ISI conditions as the amplitudes of the actual bimodal stimulation, and compared these with the sum of the waveforms obtained in the control IES and MTS conditions (Gondan and Röder, 2006; Hsieh et al., 1995; Torquati et al., 2003; Wühle et al., 2011). Since the MTS was delivered later than the IES in all ISI conditions, we shifted the waveform of the control MTS by $+40,60$, or $80 \mathrm{~ms}$ to simulate these conditions before summation of waveforms. We used the following equation to calculate the interaction ratio (IR) converting into percentage. This allowed us to express the degree of amplitude reduction that occurred in each bimodal condition (Ishibashi et al., 2000):

$I R(I S I)=\frac{\overline{d_{S P M}(c I E S)}+\overline{d_{S P M}(c M T S)}-\overline{d_{S P M}(B i)}}{\overline{d_{S P M}(c I E S)}+\overline{d_{S P M}(c M T S)}} \times 100$

where $C I E S, c M T S$ and $B i$ denote the control and bimodal conditions ( $B i$ signifies the $0-, 40-, 60-$, or $80-\mathrm{ms}$ ISI condition), and terms that are parenthesized and include $d_{S P M}$ with an overbar represent the averaged dSPM value in a 50-ms time window centered at a peak latency in each condition (see Results for a detailed account of how we set the timewindow of interest).

\section{Statistical analysis}

Originally, to determine the optimal ISI for cortical pain modulation, we evaluated the difference in IRs among 40-, 60-, and 80-ms ISI conditions using analysis of variance (ANOVA) with a randomized block design and stimulus condition as a factor. We compared the center coordinates of the control IES and control MTS conditions using ANOVA with a randomized block design and stimulus modality as a factor. To analyze the difference in VAS, we used an ANOVA with a randomized block design and stimulus condition as a factor. An arbitrary contrast was analyzed for evaluation of the tactile-induced analgesic effect by applying contrast coefficients $(1,-0.5,-0.5)$ for each condition (control IES, 0-ms ISI, 60-ms ISI, respectively). We employed an ANOVA with a randomized block factorial design and two factors; stimulus condition (0-ms ISI or 60-ms ISI) and measurement method (actual bimodal or summed), to evaluate source wave amplitudes. The least square means were used in Tukey's Honestly Significant Difference post-hoc test for VAS and the source wave amplitudes. We evaluated the difference in IRs between the 0 -ms ISI and 60-ms ISI conditions using a paired $t$-test. All data are mean \pm standard error (SE), and $P<0.05$ was considered significant. 


\section{Results}

Physiological measures and behavioral results

SEFs elicited by IES stimulation were recorded in three subjects in a separate experiment to confirm selective stimulation of A $\delta$ fibers. The estimated CV based on peak latencies ( $149 \pm 14.2 \mathrm{~ms}$ and $98 \pm$ $6.2 \mathrm{~ms}$ for the right dorsal hand and shoulder, respectively) and distances (mean distance; $56 \pm 4 \mathrm{~cm}$ ) in the three subjects was $11.5 \pm$

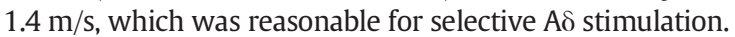

The mean VAS ratings for the control IES, 0-ms ISI, and 60-ms ISI conditions were $22.5 \pm 3.2,16.2 \pm 2.4$, and $16.6 \pm 2.6$, respectively. We found a significant main effect of stimulus condition $(F(2,20)=$ $11.7 ; P<0.001$ ), and the arbitrary contrast revealed a significant difference among the control IES and the two bimodal conditions (i.e., 0 -ms ISI and 60-ms ISI) $(\mathrm{F}(1,20)=23.3$; $P<0.001)$. This implied that the MTS had an inhibitory effect on subjective pain ratings in both bimodal conditions. There was no significant difference between the two bimodal conditions, indicating that the magnitudes of reduction in the VAS ratings for the two conditions were not statistically different.

\section{Comparison of different ISI settings}

Fig. 3 shows the temporal profile of mean source waveforms $(n=7)$ elicited by actual bimodal stimulation (blue) and summed control responses (red) in three different ISI settings (40-ms (a), 60-ms (b), and 80-ms (c)), and related statistical results (d) in Exp. I. Pain inhibition was observed in the 40-ms and 60-ms conditions, but was not evident in the 80-ms ISI condition. In addition, SEs in the 80-ms ISI condition (Figs. 3c and d) showed large inter-subject variability. We were also unable to observe pain inhibition in three out of seven subjects. Accordingly, IRs were similar between the 40- and 60-ms conditions (30.0 $\pm 9.2 \%$ and $26.0 \pm 9.4 \%$, respectively, see also Fig. 3d), but IRs were considerably smaller in the 80 -ms condition ( $5.8 \pm 18.9 \%)$. No significant main effect was found for the condition $(F(2,12)=0.76$; $P=0.49)$. Considering that the calculated difference in cortical arrival time between IESand MTS-delivered stimuli was $53 \mathrm{~ms}$, it is reasonable to assume that pain inhibition in the 40-ms ISI condition could be affected at the spinal level. Concerning the 60-ms and 80-ms ISI cortical conditions, no rationale was provided for employing the 80 -ms ISI condition because of small IRs with considerable inter-subject variability. Thus, we determined that the 60-ms ISI condition was optimal for later analysis of cortical inhibition.

\section{Cortical activation by pain and tactile stimulation}

The peak latencies for each unimodal stimulus condition in 11 subjects were $140 \pm 3.6 \mathrm{~ms}$ for the control IES and $105 \pm 2.9 \mathrm{~ms}$ for the control MTS, respectively. Fig. 4 summarizes the geometrical expression of cortical activation from control IES and MTS conditions with associated statistical results. Fig. 4a shows group-averaged data $(n=11)$ of the source activation for the control IES and control MTS conditions. The calculated center locations using Eq. (2) were $\mathrm{x}=-39.9 \pm 1.0$, $\mathrm{y}=-12.5 \pm 2.9, \mathrm{z}=17.0 \pm 1.8$ for the control IES and $\mathrm{x}=-41.7 \pm 1.4, \mathrm{y}=-20.5 \pm 2.9, \mathrm{z}=19.7 \pm 0.6$ for the control MTS. We found a significant main effect of modality $(F(1,20)=15.9$; $P<0.01)$ on the $y$-axis, while there was no significant difference on the $\mathrm{x}$-axis $(\mathrm{F}(1,20)=0.9 ; P=0.36)$ or $\mathrm{z}$-axis $(\mathrm{F}(1,20)=2.8 ; P=$ 0.13 ) (Fig. $4 \mathrm{~b}$ ). Thus, the estimated activation in the control IES condition was located more anteriorly compared with the control MTS condition. As seen in the IES condition, activity of the posterior insula was enhanced. Thus, we marked this area as R0 (Fig. 4a upper). The center coordinates of IES-evoked activation were located within R0. However, center coordinates of MTS-evoked activation were located more posteriorly, shifted toward the parietal operculum.

\section{Pain modulation of the source waveforms and MNE activity}

The suppression of pain activity by simultaneous tactile stimulation (0-ms ISI) was compared with the delay paradigm (60-ms ISI). Figs. 5a and $b$ show the temporal profile of the mean source waveforms elicited by actual bimodal stimulation (blue) and summed control responses (red). The color-matched transparent areas represent the positive and the negative SEs of the mean. As shown in Figs. $5 a$ and b, the peak responses of the group-averaged data were reduced by bimodal stimulation. Moreover, the small SEs suggested low variability across subjects. We compared the mean amplitudes of the summed source waves with those obtained from actual bimodal stimulation within the separate time windows (Figs. 5a and b, gray shadings), because peak latencies of the group-averaged data in both conditions were shifted slightly. A significant main effect of the measurement method ( $F$ (1, $30)=17.3 ; P<0.001$ ) was found, which indicated that both amplitudes in the actual bimodal conditions (0-ms ISI and 60-ms ISI) were lower than those of the summed control responses where no interaction occurred. There was no significant effect of the interaction between the stimulus condition and the measurement method $(F(1,30)=0.7$; $P=0.40$ ), which implied that the two actual bimodal conditions caused suppression of the activity to the same degree. Although the IRs a) 40-ms ISI

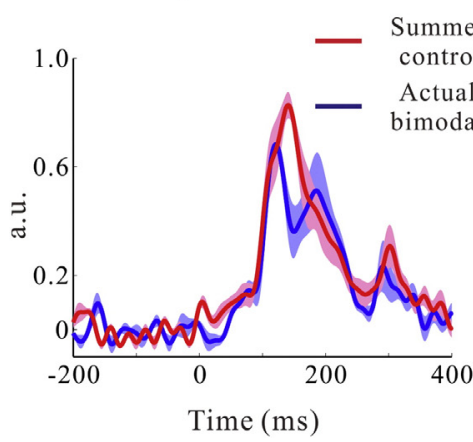

b) 60-ms ISI

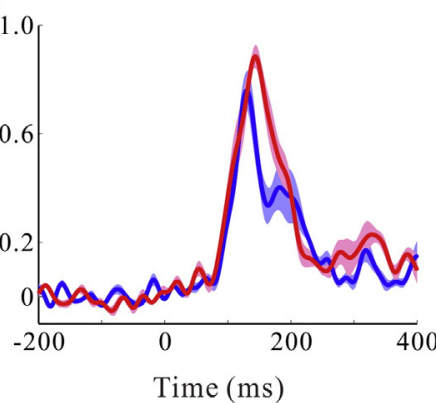

c) 80-ms ISI

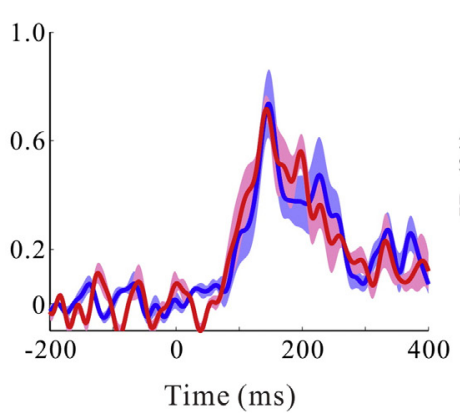

d) Comparison of the IRs

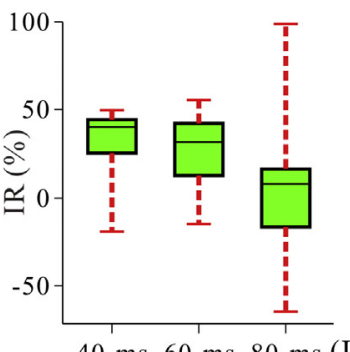

40-ms 60 -ms $80-\mathrm{ms}$ (ISI)

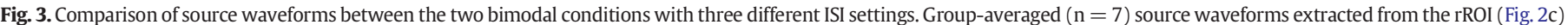

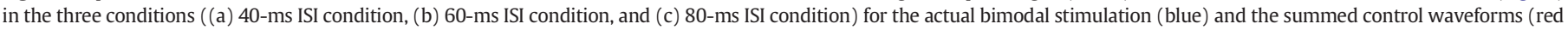

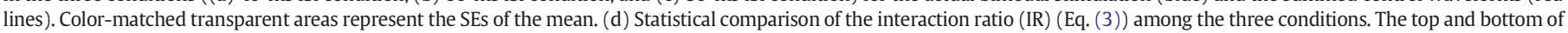
the green box represents the third and first quartiles, respectively. The red dotted lines indicate the range of minimum and maximum values. 
a) MNE activities

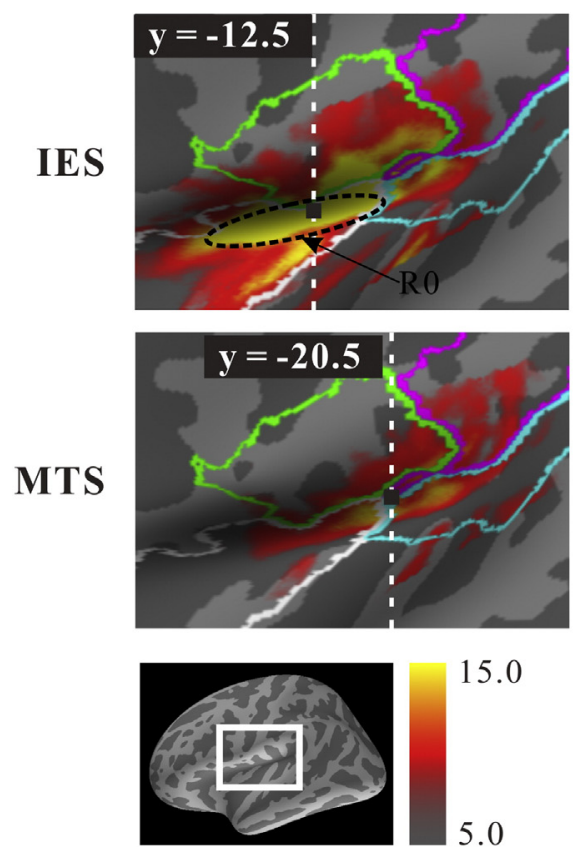

b) $3 D$ coordinates

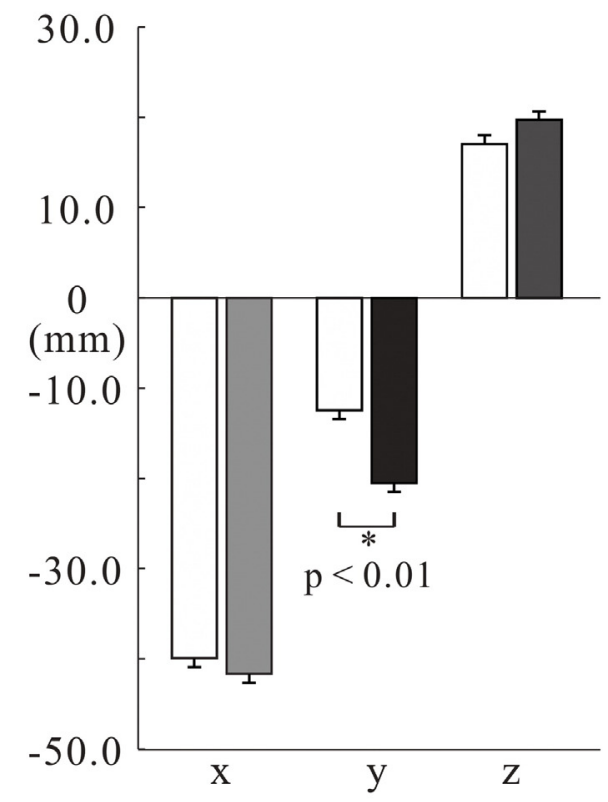

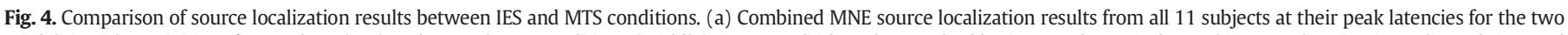

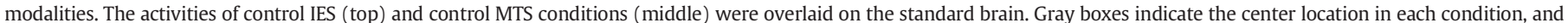

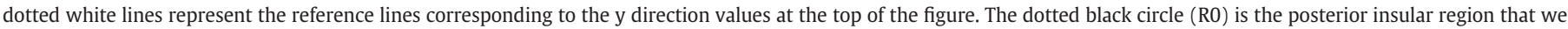

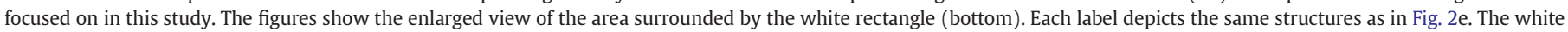

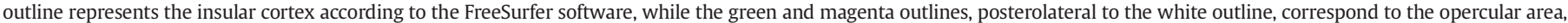

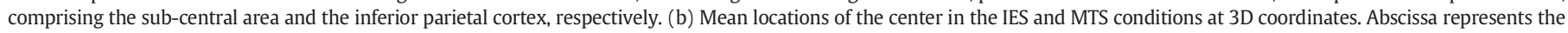
three directions ( $\mathrm{x}, \mathrm{y}$, and $\mathrm{z}$ ), while ordinate denotes the position in MNI Talairach coordinates ( $\mathrm{mm}$ ).

(Eq. (3)) showed a greater interaction in the 0 -ms ISI condition $(29.4 \pm$ $9.9 \%$ ) compared with the 60 -ms ISI condition $(16.7 \pm 9.9 \%)$, this difference was not statistically significant $(P=0.13)$. Taken together, these statistical results indicated the cortical level interaction was equipotent to the spinal level interaction in our experimental condition.

\section{Pain modulation within the IOR}

Figs. $5 \mathrm{c}$ and d show spatial profiles of group-averaged MNE results for summed control and actual bimodal conditions under the 0-ms ISI and 60-ms ISI conditions with standardization. Summed activation was located within the posterior insula (Fig. 5c-R1 and Fig. 5d-R3) and the parietal operculum (Fig. 5c-R2 and Fig. 5d-R4) in the 0-ms and 60-ms ISI conditions, and no clear difference was observed between the conditions. Consistent with source-wave amplitude reductions (Figs. 5a and b), source activation maps indicated a reduction in brain activity in the 0 -ms and 60-ms ISI conditions. In the 0-ms ISI condition, a strong reduction in activity was observed in the posterior insula (Fig. 5c-R1) and parietal operculum (Fig. 5c-R2). In the 60-ms ISI condition, reduction in activity was predominant in the posterior insula with a modest reduction in the most posterior part (Fig. 5d-R3) and the parietal operculum (Fig. 5d-R4).

\section{Discussion}

The present study clearly demonstrated pain relief by tactile input at the spinal and cortical level, showing the spatiotemporal signature of tactile-induced analgesia. Our results demonstrated the difference in cortical source locations between pain and tactile processing with modality-selective stimulation. The observed temporally-specific neural activities underpin the previous fMRI findings, which segregated multisensory representations within the IOR. Most importantly, posterior insular activity (Fig. 5d-R3) was suppressed by cortical-level pain relief.

\section{Differential representation of $A \delta$ and $A \beta$ processing in the IOR}

We found a clear functional localization of $A \delta$ and $A \beta$ inputs within the IOR. Using MNE, the two modality-specific activities were successfully identified as anterior-to-posterior distinct patterns of activation (Fig. 4a), in agreement with previous fMRI studies (Mazzola et al., 2012; zu Eulenburg et al., 2013), which consolidated differential multisensory representations within the IOR. An argument may be made that MNE is less sensitive for determining focal activities compared with other techniques such as dipole analysis or beamforming techniques. Nonetheless, the two activation clusters were clearly grouped and we could identify the center of the cluster, in accord with our previous study on laryngeal somatotopic organization (Miyaji et al., 2014). Hauk et al. (2011) also suggested that estimation of the insula using dSPM showed maximum activation around the true source location in a simulation study.

In the light of localization results, progress is required on anatomical details. Both IES and MTS-elicited activity was distributed in the proximity of the border between the posterior insula and parietal operculum (Fig. 4a), yet the x-direction showed no significant difference (Fig. 4b). Both IES and MTS are recent techniques, and detailed research, including intracranial studies, using these techniques is lacking. Thus, our study provides novel information regarding their spatial segregation. IES localization results were within the range evaluated by an intracranial recording of laser-evoked potentials (Frot et al., 2014), while MTS was localized deeply within the parietal operculum (Eickhoff et al., 2006a). Unlike electrical A $\beta$ stimulation, MTS induces natural touchlike sensations, but weaker evoked responses. This difference has a significant influence on source localization accuracy. In fact, single-dipole 
a) Source waveforms (0-ms ISI)

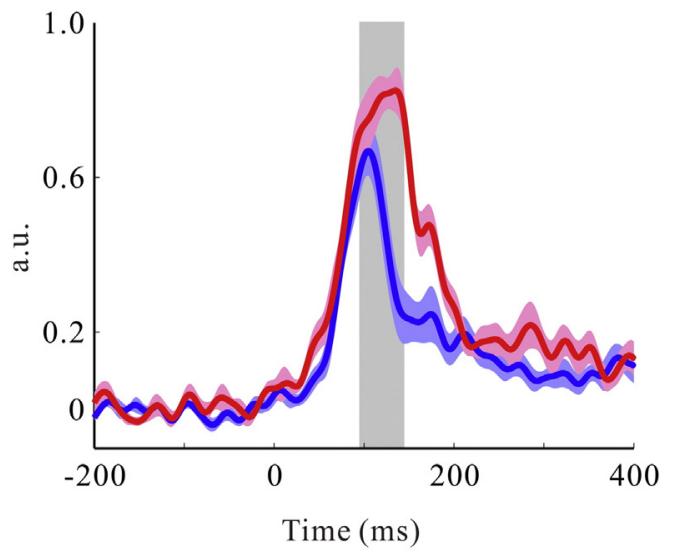

b) Source waveforms (60-ms ISI)

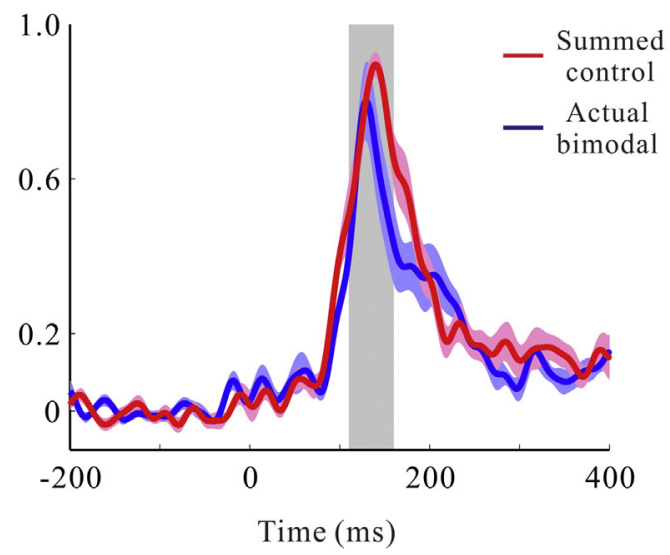

c) MNE activities at 0-ms ISI

d) MNE activities at 60-ms ISI
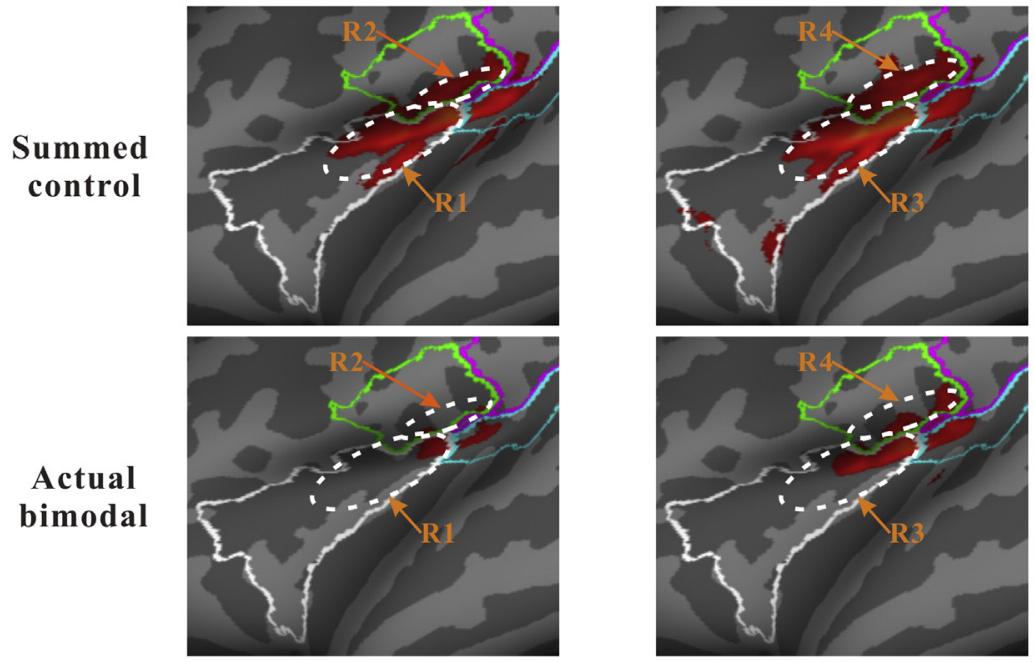

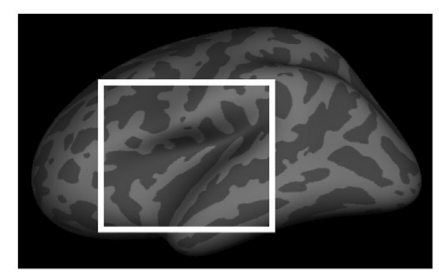

10.0

5.0

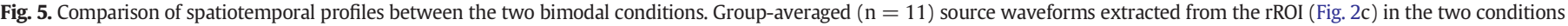

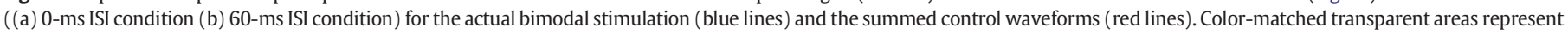

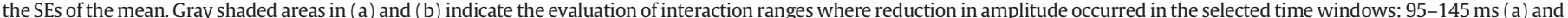

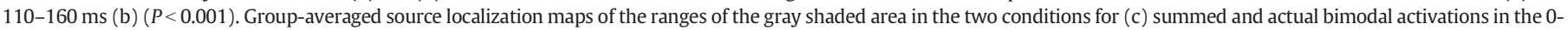

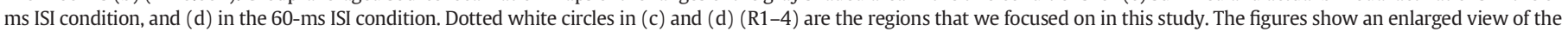
area surrounded by the white rectangle (right). Each label depicts the same structures as in Fig. 2e.

analysis could not segregate the contralateral opercular responses (Onishi et al., 2010; Onishi and Kameyama, 2011). In contrast, our MNE-based approach successfully segregated opercular activity elicited by MTS. Subsequently, we were able to clearly distinguish the source location for $A \delta-A \beta$ interactions associated with natural-touch like sensation.

\section{Temporal features of pain-tactile interaction}

On the basis of distinct spatial source localization for each stimulus modality, we investigated the timing of the pain-tactile interaction. A previous SEF study (Torquati et al., 2003) of the somatosensory gating effect demonstrated that the simultaneous presentation of painful and non-painful electric stimuli resulted in a smaller SII response compared with the sum of the responses elicited by each stimulus separately. Other studies also used the same analytical method to assess multisensory interactions with event-related potentials (e.g. Gondan and Röder, 2006). If no interference exists between the two different sensory modalities, the amplitude obtained by summation of the evoked responses to the separate-modality stimuli should be equal to that obtained by combined-modality stimulation (Hsieh et al., 1995; Ishibashi et al., 2000). To date, few studies have demonstrated that the pain-tactile interaction occurs strictly at the supraspinal level (Inui et al., 2006; Mouraux and Plaghki, 2007; Testani et al., 2015). This is likely because some of former studies did not avoid interference at the spinal cord. We arranged a time delay by adjusting the timing of tactile input to ensure nociceptive input reached the cortex earlier than tactile input, or at least simultaneously. Therefore, the amplitude difference for the bimodal stimulation condition in the present study effectively represents tactile-induced analgesia at the spinal cord and IOR. The source waveform analysis enabled us to specifically investigate the temporal features of pain modulation occurring within the IOR. The statistically significant reduction of source waveforms of bimodal stimulation was clearly observed in the group-averaged data (Figs. 5a and b). In both spinal and cortical conditions, the suppression of waveforms was observed in the 100-200 ms time window. The finding that peak latency of the bimodal source waveforms in the 0 -ms ISI condition was relatively close to peak latency in the tactile control condition suggests that most cortical activity could be elicited from tactile input, due to pain inhibition at the spinal cord level.

In contrast, the peak latency of the bimodal stimulation condition in the 60-ms condition (Fig. 5b) was approximately $150 \mathrm{~ms}$, which differed from latencies obtained from the tactile control condition. If 
nociceptive inputs were inhibited at the spinal cord (i.e., overridden by tactile input), the peak latency for the 60 -ms ISI condition could be the same as the 0 -ms ISI condition; shifted $+60 \mathrm{~ms}$ rightward, i.e., around $185 \mathrm{~ms}$. In short, nociceptive input predominates when cortical processing is initiated, and after this period, activity begins to be inhibited. Based on this assumption, it can be concluded that peak latency for the 60-ms ISI condition reflects integration of pain and tactile information in the posterior insula, whereas reduction in nociceptive input with less cortical modulation occurs in the 0-ms ISI condition.

\section{Spatial features of pain-tactile interaction}

In addition to the temporal characteristics of pain modulation, the difference in spatial features between the spinal and cortical suppression of pain were clearly observed within the IOR (Figs. $5 \mathrm{c}$ and d). Torquati et al. (2003) reported a suppressive effect for bimodal (painful and non-painful) simultaneous stimulations in the contralateral SII region. The authors suggested that the spatial overlap in nociceptive and non-nociceptive neural populations of SII could explain this effect. In addition, they argued that the functional connection between pain and tactile neuronal populations should be considered an inter-modality integrative effect in SII. In the present study, the source localization results of the 60-ms ISI condition showed clear suppression of activity in the posterior insula (see comparison of R3 in Fig. 5d), which suggests involvement of the posterior insula in nociceptive and non-nociceptive integration, i.e., cortical pain modulation.

The insula plays a key role in pain-related brain activity; however, the anterior and posterior insula have been postulated to behave differentially. Several lines of evidence support the importance of the posterior insula for sensory aspects of pain processing (Iannetti et al., 2005; Oertel et al., 2012; Timmermann et al., 2001; zu Eulenburg et al., 2013). Direct recording from the insular cortex using stereotactic electrode implantation also proved that sensory information processing is predominant in the posterior insula (Frot et al., 2007, 2014; Isnard et al., 2011). In contrast, the anterior insula encodes emotionalaffective qualities of pain (Gu et al., 2012; Singer et al., 2004). It is likely that the equivocal activation of the anterior insula compared with the posterior insula is due to the relatively weak pain intensity in our experimental design, by which an aversive response was not induced. Specifically, our pain stimuli delivered a pain sensation without unpleasantness. Thus, along with the role of the posterior insula in cortical pain modulation, our findings mainly represent sensory interaction in quantitatively- and qualitatively-discriminative aspects of pain rather than affective aspects.

\section{Spinal and cortical mechanisms of tactile-induced analgesia}

The multireceptive, i.e., wide dynamic range (WDR), neurons in the dorsal horn of the spinal cord receive information from both nociceptive and non-nociceptive inputs, and are believed to be involved in spinal level pain modulation (Le Bars, 2002; Mancini et al., 2014; Salter and Henry, 1990). IES input should attenuate at the spinal cord due to reduced firing of shared WDR neurons, and therefore, only tactileelicited impulses reached the cortex in the 0-ms ISI condition. Given that most IES input was attenuated at the spinal cord as a result of tactile-induced inhibition, such spinal-level inhibition may be more effective than cortical-level inhibition. We found that source amplitude decreased with decreasing VAS scores in the 0-ms ISI condition. However, no significant difference between the spinal and cortical pain-relief conditions was found. One possible explanation for this is that the relatively weak pain intensity, as demonstrated by lower VAS scores, resulted in a modest analgesic effect at the spinal level.

As discussed above, spatial overlap of the convergent neural population in the posterior insula could be a candidate mechanism underlying cortical pain modulation. The existence of a convergent area for multisensory processing is also suggested by an intracerebral recording study that compared the spatial distribution of laser-SEPs with those of electrical SEPs in the insular-opercular cortex (Frot et al., 2001). The posterior insula, particularly the granular area (Ig), has both anatomical and functional connections with SII and limbic areas, and the SII-Iglimbic pathway is important for both pain and tactile processing (Augustine, 1996; Friedman et al., 1986; Schneider et al., 1993). In addition to this pathway, the insula receives direct input from the ventral medial thalamic nucleus (VMpo), which is involved in the cortical processing of pain (Willis et al., 2002). Moreover, single-unit recording studies in monkeys have also identified WDR neurons within the insula (Robinson and Burton, 1980; Zhang et al., 1999), and the Ig has been recognized as one of the main regions containing this type of neuron (Robinson and Burton, 1980). Because the insula projects directly to the VMpo, it is possible that the posterior insula, rather than SII, predominates in inter-modality integration. As shown in our result, the localized brain area, i.e., the posterior insula, could further consolidate convergent pain and tactile processing with the interaction between the two modalities. Thus, the present study suggests that the posterior insula is an important site for pain inhibition by tactile input.

\section{Limitations of this study}

We limited stimulus intensity to less than $0.3 \mathrm{~mA}$ to minimize contamination with $A \beta$ inputs from IES stimulation (Inui and Kakigi, 2012; Mouraux et al., 2010). This enabled us to independently evaluate modality-specific interactions, albeit with relatively low VAS ratings. We conducted VAS ratings in a separate session to reduce the length of already long experiments, which may have led to a decrease in the concentration of the subjects. A simultaneous recording of MEG and behavioral data is a methodological option we will consider in future studies, as this would enable better quantification of the causal relationship between source amplitude reduction and subjective pain relief.

Ipsilateral responses were not analyzed in the present study. Although previous EEG/MEG studies analyzed bilateral responses (Inui et al., 2003; Lim et al., 2012; Timmermann et al., 2001), contralateral activity is generally more reproducible (Mima et al., 1998; Peyron et al., 2002). This was partly true in the present study, as ipsilateral painrelated activity was not clearly obtained and had considerable amplitude variability. Consequently, we focused on consistent contralateral responses to analyze the pain inhibitory process, while minimizing inter- and intra-individual variability.

\section{Conclusion}

Pain-tactile interaction at the cortical level was investigated using carefully timed sensory modality-specific stimuli, in combination with cortical source-based neuromagnetic analysis. We were able to clearly distinguish source location from pain and tactile inputs associated with natural-touch like sensation. Our study clearly demonstrates that the spatial feature of cortical modulation by nociceptive input was different compared with spinal modulation. The current study also provides new spatiotemporal evidence that the posterior insula is a critical area of the cortical mechanisms underlying tactile-induced analgesia in humans. Therefore, our findings contribute to the detailed electrophysiological exploration of central mechanisms underlying crossmodal sensory interactions.

\section{Acknowledgments}

This study was supported in part by a Grant-in-aid for Scientists (Project No. 25870511) from the Ministry of Education, Culture, Sports, Science, and Technology in Japan. We would like to thank Associate Professor Junji Kishimoto (Department of Research and Development of Next Generation Medicine, Faculty of Medical Sciences, Kyushu University, Fukuoka, Japan) for his statistical assistance. We also thank our colleague, Hisato Nakazono, MSc, for his help in creating the figures. 


\section{References}

Apkarian, A.V., Bushnell, M.C., Treede, R.D., Zubieta, J.K., 2005. Human brain mechanisms of pain perception and regulation in health and disease. Eur. J. Pain 9, 463-484.

Augustine, J.R., 1996. Circuitry and functional aspects of the insular lobe in primates including humans. Brain Res. Rev. 22, 229-244.

Baumgärtner, U., Treede, R.D., 2009. Are there nociceptive-specific brain potentials? J. Neurophysiol. 102, 3073-3074

Collins, D.L., Neelin, P., Peters, T.M., Evans, A.C., 1994. Automatic 3D intersubject registration of MR volumetric data in standardized Talairach space. J. Comput. Assist. Tomogr. $18,192-205$.

Dale, A.M., Sereno, M.I., 1993. Improved localization of cortical activity by combining EEG and MEG with MRI cortical surface reconstruction: a linear approach. J. Cogn. Neurosci. 5, 162-176.

Dale, A.M., Fischl, B., Sereno, M.I., 1999. Cortical surface-based analysis. I. Segmentation and surface reconstruction. NeuroImage 9, 179-194.

Dale, A.M., Liu, A.K., Fischl, B.R., Buckner, R.L., Belliveau, J.W., Lewine, J.D., Halgren, E., 2000. Dynamic statistical parametric mapping: combining fMRI and MEG for highresolution imaging of cortical activity. Neuron 26, 55-67.

Eickhoff, S.B., Amunts, K., Mohlberg, H., Zilles, K., 2006b. The human parietal operculum. Il. Stereotaxic maps and correlation with functional imaging results. Cereb. Cortex 16, 268-279.

Eickhoff, S.B., Schleicher, A., Zilles, K., Amunts, K., 2006a. The human parietal operculum. I. Cytoarchitectonic mapping of subdivisions. Cereb. Cortex 16, 254-267.

Fischl, B., Sereno, M.I., Dale, A.M., 1999. Cortical surface-based analysis. II: inflation, flattening, and a surface-based coordinate system. Neurolmage 9, 195-207.

Friedman, D.P., Murray, E.A., O'Neill, J.B., Mishkin, M., 1986. Cortical connections of the somatosensory fields of the lateral sulcus of macaques: evidence for a corticolimbic pathway for touch. J. Comp. Neurol. 252, 323-347.

Frot, M., Faillenot, I., Mauguière, F., 2014. Processing of nociceptive input from posterior to anterior insula in humans. Hum. Brain Mapp. 35, 5486-5499.

Frot, M., Garcia-Larrea, L., Guénot, M., Mauguière, F., 2001. Responses of the supra-sylvian (SII) cortex in humans to painful and innocuous stimuli. A study using intra-cerebra recordings. Pain 94, 65-73.

Frot, M., Magnin, M., Mauguière, F., Garcia-Larrea, L., 2007. Human SII and posterior insula differently encode thermal laser stimuli. Cereb. Cortex 17, 610-620.

Gondan, M., Röder, B., 2006. A new method for detecting interactions between the senses in event-related potentials. Brain Res. 1073-1074, 389-397.

Gu, X., Gao, Z., Wang, X., Liu, X., Knight, R.T., Hof, P.R., Fan, J., 2012. Anterior insular cortex is necessary for empathetic pain perception. Brain 135, 2726-2735.

Hämäläinen, M.S., Ilmoniemi, R.J., 1994. Interpreting magnetic fields of the brain: minimum norm estimates. Med. Biol. Eng. Comput. 32, 35-42.

Hauk, O., Wakeman, D.G., Henson, R., 2011. Comparison of noise-normalized minimum norm estimates for MEG analysis using multiple resolution metrics. Neurolmage 54 $1966-1974$

Hawker, G.A., Mian, S., Kendzerska, T., French, M., 2011. Measures of adult pain: Visual Analog Scale for Pain (VAS Pain), Numeric Rating Scale for Pain (NRS Pain), McGil Pain Questionnaire (MPQ), Short-Form McGill Pain Questionnaire (SF-MPQ), Chronic Pain Grade Scale (CPGS), Short form-36 Bodily Pain Scale (SF-36 BPS), and Measure of Intermittent and Constant Osteoarthritis Pain (ICOAP). Arthritis Care Res. 63 (Suppl. 11), S240-S252.

Hironaga, N., Hagiwara, K., Ogata, K., Hayamizu, M., Urakawa, T., Tobimatsu, S., 2014. Proposal for a new MEG-MRI co-registration: a 3D laser scanner system. Clin. Neurophysiol. 125, 2404-2412.

Hsieh, C.L., Shima, F., Tobimatsu, S., Sun, S.J., Kato, M., 1995. The interaction of the somatosensory evoked potentials to simultaneous finger stimuli in the human centra nervous system. A study using direct recordings. Electroencephalogr. Clin. Neurophysiol. 96, 135-142.

Iannetti, G.D., Zambreanu, L., Cruccu, G., Tracey, I., 2005. Operculoinsular cortex encodes pain intensity at the earliest stages of cortical processing as indicated by amplitude of laser-evoked potentials in humans. Neuroscience 131, 199-208.

Inui, K., Kakigi, R., 2012. Pain perception in humans: use of intraepidermal electrical stimulation. J. Neurol. Neurosurg. Psychiatry 83, 551-556.

Inui, K., Tran, T.D., Hoshiyama, M., Kakigi, R., 2002. Preferential stimulation of A $\delta$ fibers by intra-epidermal needle electrode in humans. Pain 96, 247-252.

Inui, K., Tran, T.D. Oiu, Y, Wang, X., Hoshiyama, M., Kakigi, R, 2003. A comparative magnetoencephalographic study of cortical activations evoked by noxious and innocuous somatosensory stimulations. Neuroscience 120, 235-248.

Inui, K., Tsuji, T., Kakigi, R., 2006. Temporal analysis of cortical mechanisms for pain relief by tactile stimuli in humans. Cereb. Cortex 16, 355-365.

Ishibashi, H., Tobimatsu, S., Shigeto, H., Morioka, T., Yamamoto, T., Fukui, M., 2000. Differential interaction of somatosensory inputs in the human primary sensory cortex: a magnetoencephalographic study. Clin. Neurophysiol. 111, 1095-1102.

Isnard, J., Magnin, M., Jung, J., Mauguière, F., Garcia-Larrea, L., 2011. Does the insula tell our brain that we are in pain? Pain 152, 946-951.

Kakigi, R., Shibasaki, H., 1991. Estimation of conduction velocity of the spino-thalamic tract in man. Electroencephalogr. Clin. Neurophysiol. 80, 39-45.

Kakigi, R., Shibasaki, H., 1992. Mechanisms of pain relief by vibration and movement. J. Neurol. Neurosurg. Psychiatry 55, 282-286.

Kanamori, Y., Shigeto, H., Hironaga, N., Hagiwara, K., Uehara, T., Chatani, H., Sakata, A. Hashiguchi, K., Morioka, T., Tobimatsu, S., Kira, J., 2013. Minimum norm estimates in MEG can delineate the onset of interictal epileptic discharges: a comparison with ECoG findings. Neuroimage Clin. 2, 663-669.

Kurth, F., Eickhoff, S.B., Schleicher, A., Hoemke, L., Zilles, K., Amunts, K., 2010. Cytoarchitecture and probabilistic maps of the human posterior insular cortex. Cereb. Cortex 20, 1448-1461.
Le Bars, D., 2002. The whole body receptive field of dorsal horn multireceptive neurones. Brain Res. Rev. 40, 29-44.

Lim, M., Kim, J.S., Chung, C.K., 2012. Modulation of somatosensory evoked magnetic fields by intensity of interfering stimuli in human somatosensory cortex: an MEG study. NeuroImage 61, 660-669.

Lundeberg, T., Nordemar, R., Ottoson, D., 1984. Pain alleviation by vibratory stimulation. Pain 20, 25-44.

Mancini, F., Nash, T., Iannetti, G.D., Haggard, P., 2014. Pain relief by touch: a quantitative approach. Pain 155, 635-642.

Mazzola, L., Faillenot, I., Barral, F.G., Mauguière, F., Peyron, R., 2012. Spatial segregation of somato-sensory and pain activations in the human operculo-insular cortex. NeuroImage 60, 409-418.

Melzack, R., Wall, P.D., 1965. Pain mechanisms: a new theory. Science 150, 971-979.

Mima, T., Nagamine, T., Nakamura, K., Shibasaki, H., 1998. Attention modulates both primary and second somatosensory cortical activities in humans: a magnetoencephalographic study. J. Neurophysiol. 80, 2215-2221.

Miyaji, H., Hironaga, N., Umezaki, T., Hagiwara, K., Shigeto, H., Sawatsubashi, M., Tobimatsu, S., Komune, S., 2014. Neuromagnetic detection of the laryngeal area: sensory-evoked fields to air-puff stimulation. NeuroImage 88, 162-169.

Molins, A., Stufflebeam, S.M., Brown, E.N., Hämäläinen, M.S., 2008. Quantification of the benefit from integrating MEG and EEG data in minimum 12-norm estimation. Neurolmage 42, 1069-1077.

Mouraux, A., Plaghki, L., 2007. Cortical interactions and integration of nociceptive and non-nociceptive somatosensory inputs in humans. Neuroscience $150,72-81$

Mouraux, A., Iannetti, G.D., Plaghki, L., 2010. Low intensity intra-epidermal electrical stimulation can activate Aס-nociceptors selectively. Pain 150, 199-207.

Oertel, B.G., Preibisch, C., Martin, T., Walter, C., Gamer, M., Deichmann, R., Lötsch, J., 2012. Separating brain processing of pain from that of stimulus intensity. Hum. Brain Mapp. 33, 883-894.

Omori, S., Isose, S., Otsuru, N., Nishihara, M., Kuwabara, S., Inui, K., Kakigi, R., 2013. Somatotopic representation of pain in the primary somatosensory cortex (S1) in humans. Clin. Neurophysiol. 124, 1422-1430.

Onishi, H., Kameyama, S., 2011. Reply to "somatosensory evoked fields by tactile-off stimulation". Clin. Neurophysiol. 122, 849

Onishi, H., Oyama, M., Soma, T., Kubo, M., Kirimoto, H., Murakami, H., Kameyama, S., 2010. Neuromagnetic activation of primary and secondary somatosensory cortex following tactile-on and tactile-off stimulation. Clin. Neurophysiol. 121, 588-593.

Peyron, R., Frot, M., Schneider, F., Garcia-Larrea, L., Mertens, P., Barral, F.G., Sindou, M., Laurent, B., Mauguière, F., 2002. Role of operculoinsular cortices in human pain processing: converging evidence from PET, fMRI, dipole modeling, and intracerebral recordings of evoked potentials. Neurolmage 17, 1336-1346.

Peyron, R., Laurent, B., García-Larrea, L., 2000. Functional imaging of brain responses to pain. A review and meta-analysis (2000). Neurophysiol. Clin. 30, 263-288.

Robinson, C.J., Burton, H., 1980. Somatic submodality distribution within the second somatosensory (SII), 7b, retroinsular, postauditory, and granular insular cortical areas of M. fascicularis. J. Comp. Neurol. 192, 193-108.

Roy, E.A., Hollins, M., Maixner, W., 2003. Reduction of TMD pain by high-frequency vibration: a spatial and temporal analysis. Pain 101, 267-274

Salter, M.W., Henry, J.L., 1990. Differential responses of nociceptive vs. non-nociceptive spinal dorsal horn neurones to cutaneously applied vibration in the cat. Pain 40, 311-322.

Schneider, R.J., Friedman, D.P., Mishkin, M., 1993. A modality-specific somatosensory area within the insula of the rhesus monkey. Brain Res. 621, 116-120.

Singer, T., Seymour, B., O'Doherty, J., Kaube, H., Dolan, R.J., Frith, C.D., 2004. Empathy for pain involves the affective but not sensory components of pain. Science 303, 1157-1162.

Staud, R., Robinson, M.E., Goldman, C.T., Price, D.D., 2011. Attenuation of experimental pain by vibro-tactile stimulation in patients with chronic local or widespread musculoskeletal pain. Eur. J. Pain 15, 836-842.

Taulu, S., Kajola, M., Simola, J., 2004. Suppression of interference and artifacts by the signal space separation method. Brain Topogr. 16, 269-275.

Testani, E., Le Pera, D., Del Percio, C., Miliucci, R., Brancucci, A., Pazzaglia, C., De Armas, L., Babiloni, C., Rossini, P.M., Valeriani, M., 2015. Cortical inhibition of laser pain and laser-evoked potentials by non-nociceptive somatosensory input. Eur. J. Neurosci. 42, 2407-2414.

Timmermann, L., Ploner, M., Haucke, K., Schmitz, F., Baltissen, R., Schnitzler, A., 2001. Differential coding of pain intensity in the human primary and secondary somatosensory cortex. J. Neurophysiol. 86, 1499-1503.

Torquati, K., Pizzella, V., Della Penna, S., Franciotti, R., Babiloni, C., Romani, G.L., Rossini, P.M., 2003. "Gating” effects of simultaneous peripheral electrical stimulations on human secondary somatosensory cortex: a whole-head MEG study. NeuroImage 20, 1704-1713.

Willis, W.D., Zhang, X., Honda, C.N., Giesler, G.J., 2002. A critical review of the role of the proposed VMpo nucleus in pain. J. Pain 3, 79-94.

Wühle, A., Preissl, H., Braun, C., 2011. Cortical processing of near-threshold tactile stimuli in a paired-stimulus paradigm - an MEG study. Eur. J. Neurosci. 34, 641-651.

Yarnitsky, D., Kunin, M., Brik, R., Sprecher, E., 1997. Vibration reduces thermal pain in adjacent dermatomes. Pain 69, 75-77.

Zhang, Z.H., Dougherty, P.M., Oppenheimer, S.M., 1999. Monkey insular cortex neurons respond to baroreceptive and somatosensory convergent inputs. Neuroscience 94 , 351-360.

zu Eulenburg, P., Baumgärtner, U., Treede, R.D., Dieterich, M., 2013. Interoceptive and multimodal functions of the operculo-insular cortex: tactile, nociceptive and vestibular representations. NeuroImage 83, 75-86. 